\title{
Path Planning for Unmanned Underwater Vehicle Based on Improved Particle Swarm Optimization Method
}

\author{
https://doi.org/10.3991/ijoe.v14i12.9227 \\ Jianhua Xu \\ Northwestern Polytechnical University, Xi'an, Shaanxi, China \\ Sanjiang University, Nanjing, Jiangsu, China \\ $\mathrm{Hao} \mathrm{Gu}$ \\ Northwestern Polytechnical University, Xi'an, Shaanxi, China \\ Hongtao Liang $(\bowtie)$ \\ Shaanxi Normal University, Xi'an, Shaanxi, China \\ lhtesnnu.edu.cn
}

\begin{abstract}
Path planning of Unmanned Underwater Vehicle (UUV) is of considerable significance for the underwater navigation, the objective of the path planning is to find an optimal collision-free and the shortest trajectory from the start to the destination. In this paper, a new improved particle swarm optimization (IPSO) was proposed to process the global path planning in a static underwater environment for UUV. Firstly, the path planning principle for UUV was established, in which three cost functions, path length, exclusion potential field between the UUV and obstacle, and attraction potential field between UUV and destination, were considered and developed as an optimization objective. Then, on the basis of analysis traditional particle swarm optimization (PSO), the timevarying acceleration coefficients and slowly varying function were employed to improve performance of PSO, time-varying acceleration coefficients was utilized to balance the local optimum and global optimum, and slowly varying function was introduced into the updating formula of PSO to expand search space and maintain particle diversity. Finally, numerical simulations verify that, the proposed approach can fulfill path planning problems for UUV successfully.
\end{abstract}

Keywords-Unmanned Underwater Vehicle, path planning, PSO, time-varying acceleration coefficient, slowly varying function

\section{$1 \quad$ Introduction}

With the popularity and wide application of UUV (Unmanned Underwater Vehicle) in the ocean engineering and military operation fields, UUV as an indispensable intelligent navigation vehicle has attracted many attentions [1-4]. The path planning of UUV is one of the challenging problem in the application processing, because it is a foundation to ensure safe and efficient completion of complex underwater tasks [5-10]. The main objective of the path planning is considered as the computation an optimal 
collision-free and the shortest trajectory from the start to the destination without hitting with any of the obstacles in underwater environment.

Generally, path planning problem can be classified into two categories: local path planning (LPP) and global path planning (GPP) [11,12]. LPP mainly relies on the unknown or partially known environmental information that is obtained from the acoustic sensors, such as forward-looking sonar. In dealing with the LPP problem, some approaches have been applied, such as artificial potential field method [13], fuzzy logic algorithm [14], and the rolling windows method [15]. However, those methods always exists some significant problems such as high computational cost, ineffective in path planning when the underwater space is large, and even deadlock phenomenon.

GPP as the other approach has been widely put forward, which can be formulated as a constrained optimization problem that is a non-deterministic polynomial hard problem with high computational complexity. Recent decades, numerous GPP approaches have been proposed such as diagrams algorithm and visibility graphs, especially global evolutionary algorithms [6,8,11,16-18]. The evolutionary algorithms compared with others GPP methods have advantages: easy implementation, strong capacity of global search, fast convergence rate and good robustness. Generally, there are some useful evolutionary algorithms [16] has been developed such as the heuristic A-star algorithm, genetic algorithm, ant colony algorithm, differential evolution, and obtained some great achievements.

Among those algorithm, PSO $[6,16,19]$ as a global evolutionary algorithm is inspired by the behaviors of stochastic swarm such as flocks of birds and schools of fish, which mainly uses the swarm intelligent to achieve the goal of optimization. PSO has been applied to many domains with good performance such as system identification, neural networks and system control because of its characteristics of swarm intelligence, intrinsic parallelism, and inexpensive computational. However, Due to a few adjustable parameters such as population size, inertia weight and acceleration coefficients, PSO suffered from the premature convergence and trapping in local optimum problem, and even lack of population diversity. Several improved PSO algorithms, such as PSOlinearly inertia weight, PSO-fuzzy inertia weight and PSO-nonlinear inertia weight, had been proposed $[8,19]$. At present those methods have obtained with a certain performance improvement, but convergence and scarce exploration was also the frequent problems in the application process.

Inspired by the above discussion, a new particle swarm optimization algorithm (IPSO) is developed to solve global path planning for UUV in this paper. The remainder of this paper is organized as follow. The formulation and principle of global path planning for UUV is established in the Section 2, the UUV modeling and cost function are the research focus. The Particle swarm optimization is described briefly in the Section3. Improved PSO algorithm is presented by time-varying acceleration coefficients and slowly varying function in Section 4 . Section 5 describes experimental simulation. Finally, conclusion of the study and future work in the paper are given in Section 6. 


\section{Formulation and Principle of Global Path Planning for UUV}

The goal of path planning for UUV is to find an optimal collision free and the shortest trajectory path to avoid obstacles between the start and the destination with in an unknown underwater environment. To clear illustrate the path planning problem for UUV, this section presents formulation and principle of global path planning for UUV.

\subsection{UUV Modeling and Environment Modeling}

To clearly demonstrate path planning for UUV, UUV modeling and environmental modeling are developed by three assumptions, which are shown as following.

(1) UUV model is simplified as a particle description, and its kinematic model is demonstrated as:

$$
\left\{\begin{array}{l}
\dot{x}=v \cos \theta \\
\dot{y}=v \sin \theta \\
\dot{\theta}=\omega
\end{array}\right.
$$

where $(x, y)$ denotes the position of the UUV. $\theta$ denotes the heading, and $v$ denotes the velocity.

(2) The UUV system moves on a two-dimensional space, and the motion region can be expressed by finite convex polygons, in which there are finite number of unknown static obstacles. The UUV sensor system can determine the position of the obstacle by the perceived obstacle information and estimate the obstacle size, but limited scope of perception. The detection range of UUV sensor system is a circular area with radius $\mathrm{r}$.

(3) The obstacle is depicted as the circle, which is subjected to a corresponding puffing process according to the actual dimensions and safety requirements of the UUV, that is, the radius of the obstacle includes its own radius and the radius of rotation of the UUV. Also, the obstacle boundary is a safe area.

\subsection{Cost Function Modeling}

It is very important to select a fitness function to evaluate the path individual. The tradition method of cost function generally considers the path length, which is shown as Fig.1. 


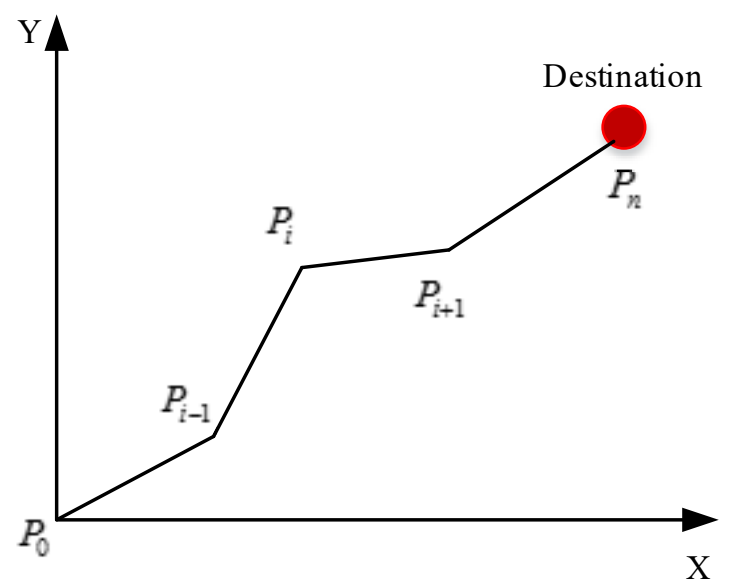

Fig. 1. Cost Function of Path Length

In Fig. $1, P_{0}\left(x_{0}, y_{0}\right)$ and $P_{d}\left(x_{d}, y_{d}\right)$ respectively denote the starting point and destination point coordinates for UUV planning, and $P_{i}\left(x_{i}, y_{i}\right)$ denotes i-th path point, $i=1,2, \cdots, n$. To simplify the planning process, UUV searches the path point $P_{i}\left(x_{i}, y_{i}\right)$ by the fixed step length $\mathrm{S}$. Thus, cost function can be calculated as follow

$$
f_{l}=\sum_{i=0}^{n} L_{P_{i}} L_{P_{i+1}}
$$

where $L_{P_{i}} L_{P_{i+1}}$ denotes the length between the point $P_{i}$ and $P_{i+1}$.

According to the Eq.(2), the path planning of UUV can be converted to find the shortest path, however the constraint, whether the line between point $P_{i}$ and $P_{i+1}$ appear in the obstacles region, is not considered, which can only be judged by the algorithm itself and increased the complexity of algorithm design and computation.

In order to overcome the above shortcoming, wo consider the attractive and repulsive fields as the other factors according to potential field theory, in which every search step onley need to find the best heading angle ${ }_{[6]}$.

(1). Cost function of repulsive potential field in avoiding obstacle. In underwater environment, there exists some static obstacle, which is shown as Fig.2. 


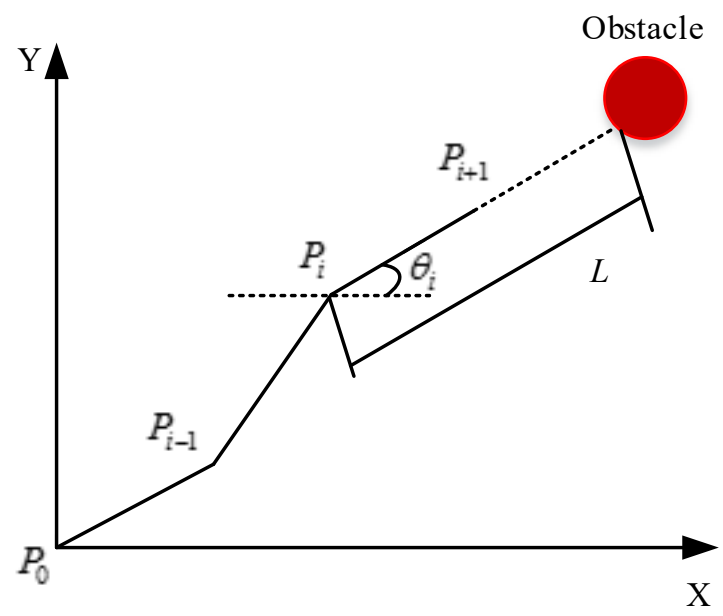

Fig. 2. Cost Function of repulsive Potential Field

In Fig.2, $P_{i}$ denotes the positon at i-th point, $P_{i+1}$ denotes the candidate position at $\mathrm{i}+1$ point, and $\mathrm{L}$ denotes the distance between the $\mathrm{i}$-th point and obstacle at the heading angle ${ }^{\theta_{i}}$. So, the cost function of avoiding obstacle can be defined as

$$
f_{o}=S / L\left(\theta_{i}\right)
$$

where $\mathrm{S}$ denotes the step length. At the heading angle $\theta_{i}$, if the distance between UUV and obstacle is larger, the cost function is greater, otherwise the cost function is smaller.

(2). Cost Function of attractive potential field to the destination. According to the potential field theory, there is an attractive potential field existing between the UUV and destination, so attraction potential field between them is shown as Fig.3. 


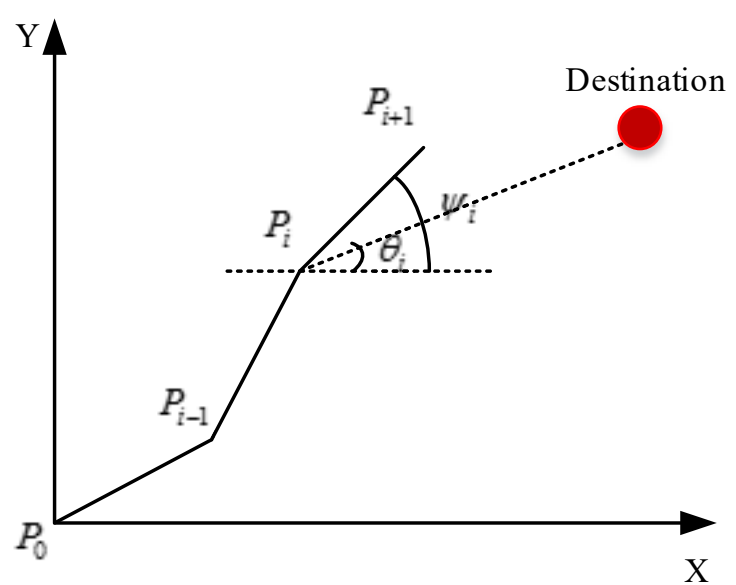

Fig. 3. Cost Function of attractive Potential Field

In Fig. $3,{ }^{\theta_{i}}$ denotes the angle between the current point $P_{i}$ and the destination point $P_{d}$, and ${ }^{{ }_{i}}$ denotes the heading angle between the candidate point $P_{i+1}$ and destination point $P_{d}$. If attractive potential field obey the normal distribution, the cost function of attractive potential field to the destination can be defined as

$$
f_{d}=\frac{1}{\sqrt{2 \pi \sigma}} e^{-\frac{(\psi-\theta)^{2}}{2 \sigma^{2}}}
$$

where $\sigma$ is parameter of normal distribution.

Then, the cost function of attractive and repulsive fields can be calculated as

$$
f_{p}=k_{o} f_{o}-k_{d} f_{d}, k_{o}>0, k_{d}>0
$$

where $k_{o}$ and $k_{d}$ are the weight coefficient.

Finally, according to the Eq.(2)and Eq.(5), total cost function of the path planning for UUV can be calculated as

$$
f=w_{l} f_{l}+w_{p} f_{p}, w_{l}>0, w_{p}>0
$$

where ${ }^{w_{l}}$ and ${ }^{w_{p}}$ are the weight coefficient.

Based on the above analysis, the cost function of path planning at the current path point is established for UUV, the IPSO is used to search the trajectory of the cost function that obtains the minimum value of path point. And searched point can be taken as optimal path point at the next navigation path. 


\section{Improved PSO algorithm}

In PSO, there exists two questions falling into local minima and lack of diversity of particles in practical application. How to solve the questions has attracted some attention. Therefore, we propose a novel Improved PSO (IPSO) with the purpose of increasing performance in this paper. In IPSO, time-varying acceleration coefficients and slowly varying function were employed, time-varying acceleration coefficients is utilized to balance the local optimum and global optimum, and slowly varying function is introduced into the updating formula of PSO to expand search space and maintain particle diversity.

\subsection{Time-Varying Acceleration Coefficients}

Acceleration coefficients determines the cognitive experience of particle and social experience of all particle in the search of swarm trajectory. That is, the larger $\mathrm{cl}$ is, the more is the attraction of particle toward its own personal best, therefore the more explorative capability. In contrast, the larger $\mathrm{c} 2$ is, the more is the attraction of particles toward swarm global best, therefore the more exploitative capability. Therefore, the appropriate balance of the control learning factors $\mathrm{c} 1$ and $\mathrm{c} 2$ is very important to find the optimal solution accurately and efficiently.

Ideally, at the early stages of the optimization processing, particles should be focus more on exploration of region of search space in hope of finding the region included global optimum. And at the later of the optimization processing, particles should be focus more on exploitative and converge towards the global optimum. Therefore, to meet the requirement of ideal acceleration coefficients, $\mathrm{c} 1$ and $\mathrm{c} 2$ are updated in a way that the cognitive part is reduced and social part is increased along iteration process. So, a new time-varying acceleration coefficients is employed to enhance exploration and exploitation of particle towards optimal solution, which are defined as [20,21]

$$
\begin{gathered}
c_{1}=c_{1, \text { ini }}+\frac{\left(c_{1, \text { fin }}-c_{1, \text { ini }}\right)}{K} k \\
c_{2}=c_{2, \text { ini }}+\frac{\left(c_{2, \text { fin }}-c_{2, \text { ini }}\right)}{K} k \\
c_{3}=c_{1} \times\left(1-e^{\left(-c_{2} \times k\right)}\right)
\end{gathered}
$$

where $\mathrm{c}$, ini and $\mathrm{c} 2$, ini are the initial values of cognitive factor and social factor respectively, c1, fin, and c2, fin are the final values of cognitive factor and social factor respectively. ${ }^{c_{3}}$ as the adding part in Eq.(7) is utilized to converge to a better solution due to the fact that $C_{\text {best }}^{k}$ the best solution in the k iteration. Therefore, the velocity and position of the particles are updated as: 


$$
\begin{gathered}
V_{i D}^{k+1}=w v_{i D}^{k}+c_{1} r_{1}\left(P_{b e s t}^{k}-x_{i D}^{k}\right)+c_{2} r_{2}\left(G_{b e s t}^{k}-x_{i D}^{k}\right)+c_{3} r_{3}\left(C_{b e s t}^{k}-x_{i D}^{k}\right) \\
x_{i D}^{k+1}=x_{i D}^{k}+V_{i D}^{k+1}
\end{gathered}
$$

where ${ }^{r}$ is a random number in the interval of [0,1], similar to the $\mathrm{r} 1$ and $\mathrm{r} 2$.

\subsection{Maintain particle diversity based on Slowly-Varying Function}

In order to maintain swarm diversity of and expand search space, slowly-varying function is introduced into the updating formula of PSO algorithm [19,22].

Definition1: Given function $1(\mathrm{x}), \forall \delta>0$ and a $>0$, there exists $1(\mathrm{x})>0$ in $[a,+\infty)$, if $x^{\delta} l(x)_{\text {is monotone increasing and }} x^{-\delta} l(x)$ is monotone decreasing, then $1(\mathrm{x})$ is called as the slowly varying function(SVF).

Definition2: Given SVF 1(x), $\forall \delta>0$ and a $>0$, in $[a,+\infty), x^{\delta} l(x)_{\text {is defined as }}$ regular varying function (RVF).

RVF is employed to use in early-stage in PSO optimization because of the fact that PSO need better global optimization ability in the early-stage. The update formulates are calculated as

$$
\begin{gathered}
V_{i D}^{k+1}=w v_{i D}^{k}+c_{1} r_{1}\left(P_{b e s t}^{k}-x_{i D}^{k}\right)+c_{2} r_{2}\left(G_{\text {best }}^{k}-x_{i D}^{k}\right)+c_{3} r_{3}\left(\mathrm{C}_{\text {best }}^{k}-x_{i D}^{k}\right) \\
x_{i D}^{k+1}=x_{i D}^{k}+V_{i D}^{k+1}+x^{\delta} l(x)
\end{gathered}
$$

where $\delta$ is the control parameter.

SVF is employed to use in later-stage in PSO optimization because of the fact that algorithm needs precise local searching ability. The update formulates are calculated as

$$
\begin{gathered}
V_{i D}^{k+1}=w v_{i D}^{k}+c_{1} r_{1}\left(P_{b e s t}^{k}-x_{i D}^{k}\right)+c_{2} r_{2}\left(G_{\text {best }}^{k}-x_{i D}^{k}\right)+c_{3} r_{3}\left(\mathrm{C}_{\text {best }}^{k}-x_{i D}^{k}\right) \\
x_{i D}^{k+1}=x_{i D}^{k}+V_{i D}^{k+1}+l(x)
\end{gathered}
$$

SVF had been proved convergence. So, in this paper, we select a slowly varying function with increasing speed and disturbance relatively faster, it is shown as [19]

$$
l(x)=(10 \ln x)^{\gamma}
$$

where $\gamma_{\text {is the control factor. }}$ 


\subsection{Algorithm description}

Based on the above analysis and discussion, solution procedures of the proposed method are summarized as follows:

Step1: Initialize the related parameters such as the size of the population M, learning factors $\mathrm{c} 1, \mathrm{c} 2$ and $\mathrm{c} 3$, maximum number of iterations $\mathrm{K}$. initial values of cognitive coefficient $\mathrm{c} 1$, ini and $\mathrm{c}$, ini, final values of cognitive coefficient $\mathrm{c} 1$, fin, and $\mathrm{c} 2$, fin.

Step2: Initialize the particles' velocities V0 and positions X0. optimal solution of individual particle Pbest $(0)$ and particle population optimal solution Gbest $(0)$, and particle best solution iteration Cbest $(0)$.

Step3: At $\mathrm{k}=\mathrm{K}$, calculate time-varying acceleration coefficients $\mathrm{c} 1, \mathrm{c} 2$ and $\mathrm{c} 3 \mathrm{ac}-$ cording to Eq.(11)- Eq.(13).

Step4: Calculate nonlinear inertia weight according to Eq.(10). If nonlinear inertia weight is equal to the 0.5 , then turn to Step5, and If nonlinear inertia weight is smaller than 0,5 , then turn to Step6, other then turn to Step7.

Step5: Update particles' velocities Vk and positions Xk according to Eq.(14)-(15), then turn to Step8.

Step6: Update particles velocities Vk and positions $\mathrm{Xk}$ based on regular varying function according to Eq.(16)-(17), then turn to Step8.

Step7: Update particles velocities $\mathrm{Vk}$ and positions $\mathrm{Xk}$ based on slowly varying function according to Eq.(18)-(19), then turn to Step8.

Step8: calculate cost function $f$ in Eq.(6), and update the optimal solution of individual particle Pbest, particle population optimal solution Gbest, and particle best solution in the $\mathrm{k}$ iteration Cbest.

Step9: If the termination criterion is satisfied, export result of the algorithm, and iteration is equal to maximum number $\mathrm{K}$, stop the algorithm. Otherwise, $\mathrm{k}=\mathrm{k}+1$, go back to Step3.

\section{Simulation Experiment Analysis}

To verify the correctness and validity of the proposed IPSO for UUV path planning, a representative scenario simulation is implemented in this paper. Assume that the UUV avoids underwater static obstacles to reach the destination. Simulation environment is a $10000(\mathrm{~m}) \times 10000(\mathrm{~m})$ underwater space, there have eight randomly obstacles in space, which is described as circular area (A\#,B\#,C\#,D\#,E\#,F\#,G\#,H\#), and the location and radius are described as Table 1. Moreover, the size of the UUV is a circle, movement velocity is set as $2.5 \mathrm{~m} / \mathrm{s}$, the initial heading is set as $\theta=45^{\circ}$, and the sensor ranger is set as $1000 \mathrm{~m}$, the starting and destination are located at $(0,0)$ and $(10000,10000)$, respectively. The IPSO parameters are setting as population $M=200$, maximum number of iterations $\mathrm{K}=200$, maximum weight $w_{\max }=10$ and minimum weight $w_{\min }=1$.The simulation experiment is designed by using the MATLAB on PC computer, and the configuration is Intel(R) Core ${ }^{\mathrm{TM}}$ i7 CPU3.40GHz, 8.0G RAM memory. The path planning trajectory of IPSO and PSO is shown as Fig.5-Fig.6. 
As shown in Fig.5-Fig.6, the path planning of the proposed IPSO can avoid the static underwater obstacles successfully from the start to the destination, and the path is much smoother compared with PSO. That is because that the IPSO can converge faster to the global optimum and avoid falling into local optimum in the iterative process. In order to verity the better performance of the proposed method in path planning for UUV, the experiment is testified by Monte Carlo simulation of 100 times by the performance indicators: path length and run time under the same condition. The statistical results quantitative indicators of IPSO and PSO are shown in Table 2.

Table 1. The location and radius of obstacles

\begin{tabular}{|c|c|c|c|c|c|}
\hline Obstacle & Location & Radius & Obstacle & Location & Radius \\
\hline A\# & $(3250,3500)$ & 900 & E\# & $(5500,2000)$ & 900 \\
\hline B\# & $(2300,900)$ & 600 & F\# & $(5200,6600)$ & 1300 \\
\hline C\# & $(1800,7500)$ & 1200 & G\# & $(7000,5000)$ & 800 \\
\hline D\# & $(8000,7600)$ & 600 & H\# & $(8500,2540)$ & 800 \\
\hline
\end{tabular}

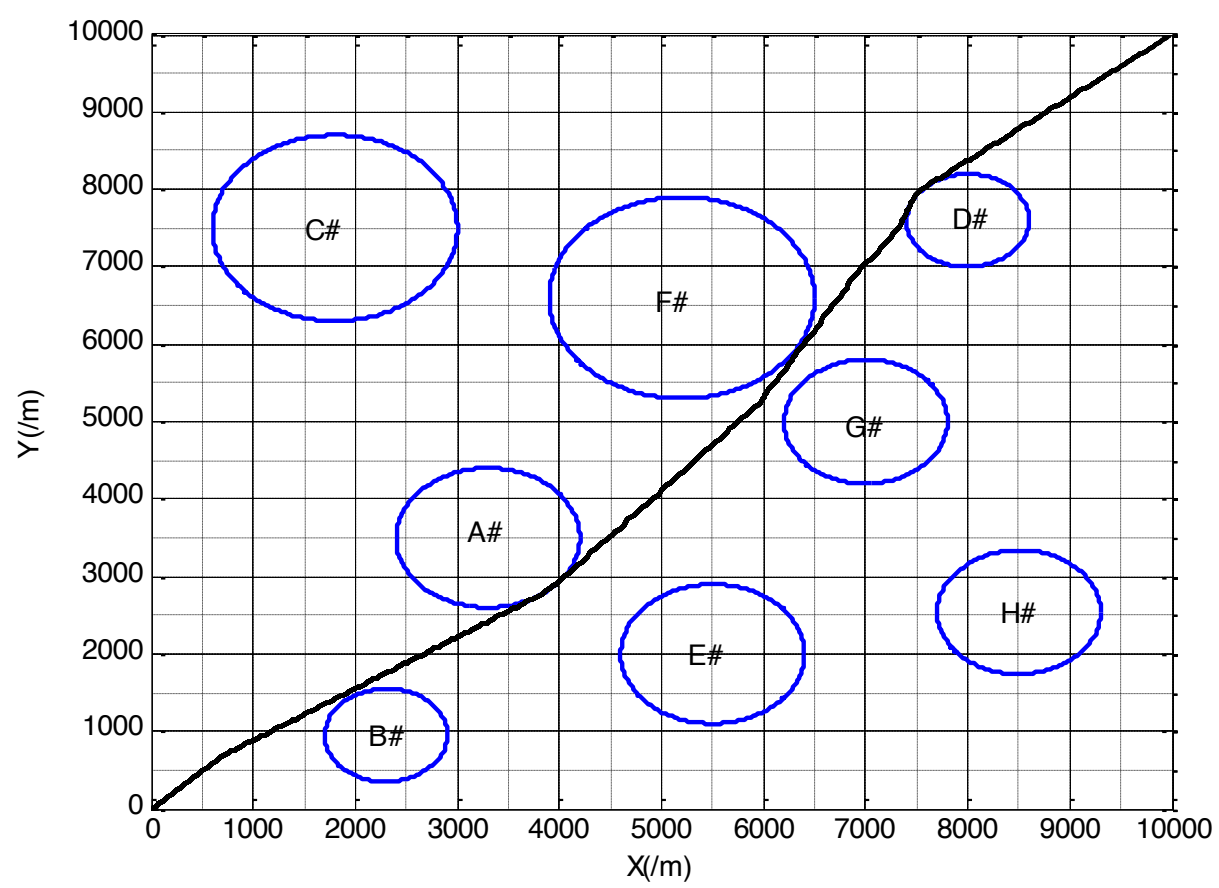

Fig. 4. Path planning trajectory of IPSO 


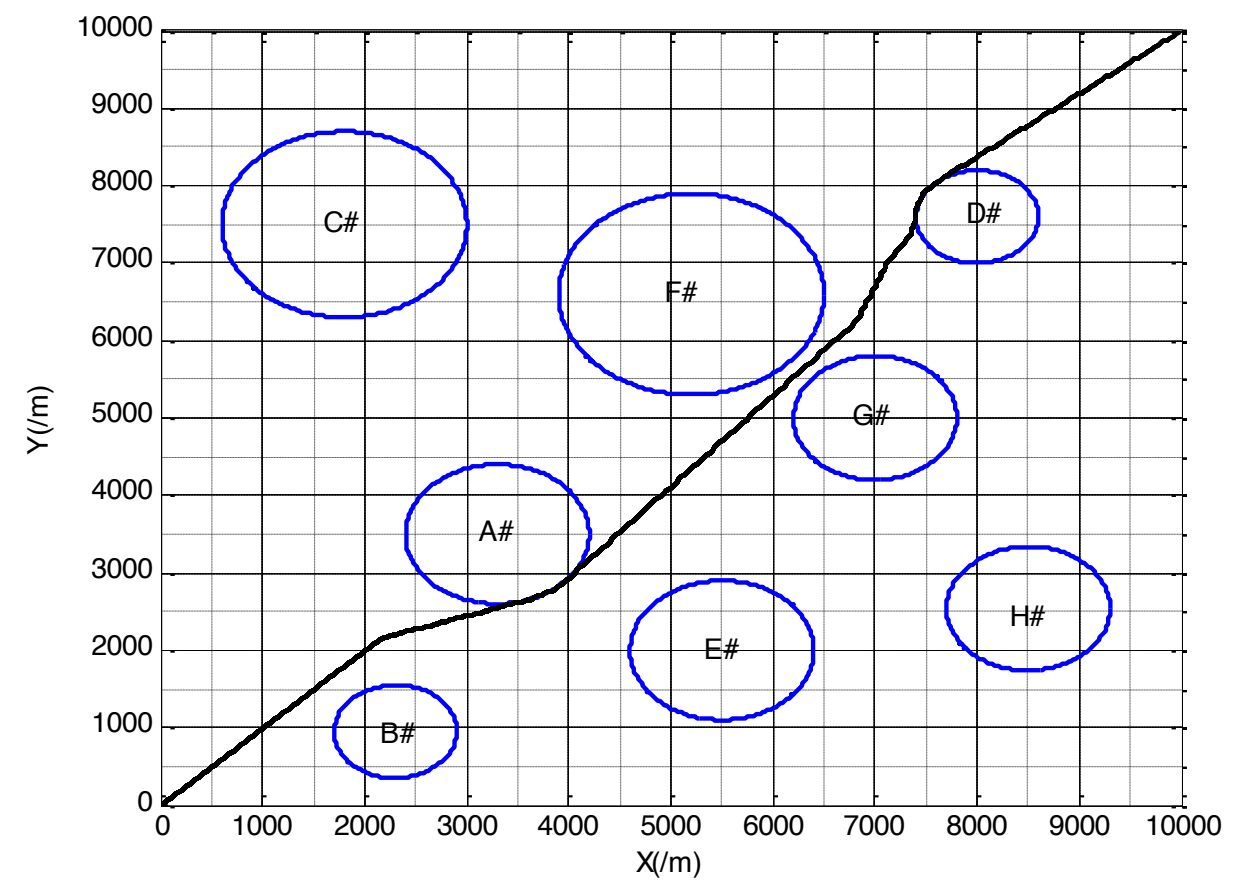

Fig. 5. Path planning trajectory of PSO

Table 2. Comparison results on path length and run time

\begin{tabular}{|c|c|c|}
\hline Methods & Path Length & Run Time \\
\hline PSO & $16142(\mathrm{~m})$ & $6456.8(\mathrm{~s})$ \\
\hline IPSO & $14980(\mathrm{~m})$ & $5992.0(\mathrm{~s})$ \\
\hline
\end{tabular}

As can be seen from Table 2, it is reported that path length and run time with our proposed method reduces respectively by all 7.2\% compared with PSO under the same simulation condition. Obviously, the proposed IPSO has a better performance in path planning for UUV.

\section{Conclusions and Future Work}

The path planning of UUV is one of challenging problem, because it is a foundation to ensure safe and efficient completion of underwater complex tasks. In this paper, a new improved particle swarm optimization (IPSO) is proposed to find a collision-free and the shortest path from a start to a destination without hitting with any of the obstacle. There are several contributions in this method as follows: (1) path planning principle for UUV is established, in which three cost functions, path length, attractive and repulsive fields are developed as an optimization objective. And (2) time-varying acceleration coefficients and slowly varying function are employed to improve 
performance of IPSO. To verify the feasibility and effectiveness of proposed method, compared experiment has been established. Simulation results demonstrate that the IPSO can effectively fulfill path planning for UUV. Our future work will focus on the exact application of our proposed method in re-planning and navigation controller design, and multiple UUV formation coordinated control is also another hot issue in this field.

\section{Acknowledgment}

The work was sponsored by Qing Lan Project of Jiangsu Province.

\section{$7 \quad$ Reference}

[1] A. Spears, M. West, M. Meister, J. Buffo, C. Walker, T.R. Collins, A. Howard, B. Schmidt, Under ice in Antarctica: the Icefin unmanned underwater vehicle develop-ment and deployment, IEEE Robotics \& Automation Magazine, 23(4):30-41, 2016. https://doi.org/10.1109/ MRA.2016.2578858

[2] H.T. Liang, F.J. Kang, Tracking UUV based on interacting multiple model unscented particle filter with multi-sensor information fusion, Optik, 126(24):5067-5073,2015. https://doi.org/10.1016/j.ijleo.2015.09.062

[3] F. Valentinis, A. Donaire, T. Perez, Energy-based motion control of a slender hull unmanned underwater vehicle, Ocean Engineering, 104:604-616, 2015. https://doi.org/10.1016/j.oceaneng.2015.05.014

[4] K.L. Davies, R.M. Moore, Unmanned underwater vehicle fuel cell energy/power sys-tem technology assessment, IEEE Journal of Oceanic Engineering, 32(2):365-372, 2007. https://doi.org/10.1109/JOE.2007.893690

[5] M.B. Loc, H.S. Choi, S.S. You, T.N. Huy, Time optimal trajectory design for unmanned underwater vehicle, Ocean Engineering, 89:69-81, 2014. https://doi.org/10.1016/j.ocean eng.2014.06.019

[6] Z.P. Yan,C. Deng, Y.F. Zhao, B.Y. Li, Path planning method for UUV near sea bot-tom, Journal of Harbin Engineering University, 35(3):307-312,2014.

[7] T. Phanthong, T. Maki, T. Ura, T. Sakamaki, P. Aiyarak, Application of A* algorithm for real-time path re-planning of an unmanned surface vehicle avoiding underwater obstacles, Journal of Marine Science and Application,13(1):105-116,2014. https://doi.org/10.1007/ s11804-014-1224-3

[8] X.Z. Jia, K.S. Liu, M. Zhang, C. Deng, D. Shan, Potential Field Method for Unmanned Underwater Vehicle Path-planning, Computer Measurement \& Control, 27(1): 144-148, 2017.

[9] B. Sun, D.Q. Zhu, Y.Y. Yang, Fuzzy path planning for autonomous underwater vehi-cles based on particle swarm optimization, Chinese High Technology Letters, 23(12):1284$1291,2013$.

[10] Z.W. Wen, W.J. Cai, C.W. Yang, Three-dimensional Path Planning Method Based on Improved Ant Colony Algorithm for UUV, Torpedo Technology, 24(2):120-125, 2016.

[11] N.Y. Zeng, H. Zhang, Y.P. Chen, B.Q. Chen, Y.R. Liu, Path planning for intelligent ro-bot based on switching local evolutionary PSO algorithm, Assembly Automa-tion,36(2):120126, 2016. https://doi.org/10.1108/AA-10-2015-079 
Paper-Path Planning for Unmanned Underwater Vehicle Based on Improved Particle Swarm Optimi...

[12] E. Peymani, T.I. Fossen, Path following of underwater robots using Lagrange multipli-ers, Robotics and Autonomous Systems, 67:44-52, 2015. https://doi.org/10.1016/j.robot.2014.10.011

[13] M. Fakoor, A. Kosari, M. Jafarzadeh, Revision on fuzzy artificial potential field for humanoid robot path planning in unknown environment, International Journal of Advanced Mechatronic Systems, 6(4):174-183, 2015. https://doi.org/10.1504/IJAMECHS.2015.072707

[14] C.J. Huang, Y.W. Wang, H.M. Chen, H.W. Tsai, J.J. Jian, A.L. Cheng, J.J. Liao, Application of cellular automata and type-2 fuzzy logic to dynamic vehicle path planning, Applied Soft Computing, 19:333-342, 2014. https://doi.org/10.1016/j.asoc.2014.02.023

[15] G. Loianno, C. Brunner, G. McGrath, V. Kumar, Estimation, Control, and Planning for Aggressive Flight with a Small Quadrotor with a Single Camera and IMU, IEEE Ro-botics and Automation Letters, 2(2):404-411,2017.

[16] E.G. Tsardoulias, A. Iliakopoulou, A. Kargakos, L. Petrou, A review of global path planning methods for occupancy grid maps regardless of obstacle density, Journal of Intelligent \& Robotic Systems, 84(1-4):829-858,2016. https://doi.org/10.1007/s10846-016-0362-z

[17] T. Chi, K. Fallahi, H. Leung, C.K. Tse, A Genetic Algorithm-Inspired UUV Path Plan-ner Based on Dynamic Programming, IEEE Transactions on Systems, Man and Cyber-netics, Part C, 42(6):1128-1134, 2012.

[18] J. Kok, L.F. Gonzalez, N. Kelson, FPGA implementation of an evolutionary algorithm for autonomous unmanned aerial vehicle on-board path planning, IEEE Transactions on Evolutionary Computation, 17(2):272-281,2013. https://doi.org/10.1109/TEVC.2012.2192124

[19] H.T. Liang, F.J Kang, Adaptive mutation particle swarm algorithm with dynamic non-linear changed inertia weight, Optik,127(19): 8036-8042,2016. https://doi.org/10.1016/j.ijleo. 2016.06.002

[20] B. Mohammadi-Ivatloo, A. Rabiee, A. Soroudi, M. Ehsan, Iteration PSO with time varying acceleration coefficients for solving non-convex economic dispatch prob-lems, Electrical Power and Energy Systems, 42:508-516, 2012. https://doi.org/10.1016/j.ijepes.2012.04.060

[21] A. Safari, H. Shayeghi, Iteration particle swarm optimization procedure for economic load dispatch with generator constraints, Expert Systems with Applications, 38(5):6043-6048, 2011. https://doi.org/10.1016/j.eswa.2010.11.015

[22] V.K. Ignatjev, A.V. Nikitin, S.V. Yushanov, Parametric analysis of oscillations with slowly varying frequency, Radio physics and Quantum Electronics, 53(2):132-145, 2010. https://doi.org/10.1007/s11141-010-9209-9

\section{Authors}

Jianhua Xu is a Doctorate degree candidate of School of Marine Science and Technology, Northwestern Polytechnical University, Xi'an, Shaanxi, 710072, China; in addition, he is an Associate Professor of School of Computer Science and Engineering, Sanjiang University, Nanjing, Jiangsu, 210012, China.

Hao Gu is a Professor of School of Marine Science and Technology, Northwestern Polytechnical University, Xi'an, Shaanxi, 710072, China.

Hongtao Liang is a lecturer of School of Physics and Information Technology, Shaanxi Normal University, Xi'an, Shaanxi, 710062, China.

Article submitted 13 July 2018. Resubmitted 16 October 2018. Final acceptance 01 November 2018. Final version published as resubmitted by the authors. 